On the Foundations of Dynamics.

By Dr Peddie.

Note on a Theorem in connection with the Hessian of a Binary Quantic.

By Charles Tweedie, M.A., B.Sc.

Extension of the "Medial Section" problem (Euclid II :11, VI : 30, etc.) and derivation of a Hyperbolic Graph.

By R. E. Anderson, M.A.

To divide the straight line $\mathrm{AB}$ (containing $a$ units) at $\mathrm{C}$ so that $\mathrm{AB} \cdot \mathrm{BC}=p \cdot \mathrm{AC}^{2}$.

\title{
$\S I$.
}

By algebra, taking the positive root,

$$
\mathrm{AC}=\frac{\mathrm{AB}}{2 p}(\sqrt{4 p+1}-1)
$$

The number $p$ may therefore have any positive value, integral or fractional, and when negative cannot exceed $\frac{1}{4}$ Secondly, $\mathrm{AC}$ and $\mathrm{AB}$ are incommensurable except when $4 p+1$ is a square :e.g., if $4 p=(q-1)(q+1)$ or if $p=q(q+1), q$ being any positive integer or fraction.

To find the surd-line $\sqrt{4 p+1}$ geometrically is the heart of the problem. Euclid solves it (II : 11) when $p=1$ by I : 47, which is also used in Ex. i., ii., iii. following; but II : 14 will sometimes be easier. Since equation (1.) becomes $\mathrm{AC}=\sqrt{4 p+1}-1$, if $\mathrm{AB}=2 p$, i.e., if the unit line is $\frac{\mathrm{AB}}{2 p}$ or $\frac{\mathrm{AM}}{p}$, we construct thus :- 
(Figure 1.)

Ex. i. Solve $\mathrm{AB} . \mathrm{BC}=3 \mathrm{AC}^{2}$. Here $p=3$, surd $=\sqrt{ } 13$. Take $A K=\frac{1}{3} A M, A R=2 A K$, and $\mathrm{KS}=\mathrm{RM}$.

$\mathrm{C}$ the point required is determined by a square described on A.S.

(Figure 2.)

Ex. ii. Solve $\mathrm{AB} \cdot \mathrm{BC}=4 \mathrm{AC}^{2}$. Here $p=4$, surd $=\sqrt{ } 17$. Take $A K=\frac{1}{4} A M$ and $K S=K M$.

$\mathrm{C}$ the point required is determined by square on AS.

(Figure 3.)

Ex. iii. Solve $\mathrm{AB} . \mathrm{BC}=7 \mathrm{AC}^{2}$. Here $p=7$, surd $=\sqrt{ } 29$. Take $\mathrm{AK}=\frac{1}{7} \mathrm{AM}, \quad \mathrm{AR}=2 \mathrm{AK}, \quad \mathrm{AT}=5 \mathrm{AK}, \quad$ and $\mathrm{KS}=\mathrm{RT}$. $\mathrm{C}$ is determined by square on AS.

(Figure 4.)

Ex. iv. Solve $\mathrm{AB} . \mathrm{BC}=\mathrm{AC}^{2}$ by II $: 14$. Here $p=1$, surd $=\sqrt{ } 5$. Produce $\mathrm{AB}$ both ways till $\mathrm{AK}=\mathrm{AM}$ the unit, and $\mathrm{AH}=5 \mathrm{AM}$ $\therefore \mathrm{AR}=\sqrt{ } \mathbf{5}$ if $\perp \mathrm{AB}$ and limited by $\frac{1}{2} \odot$ on $\mathrm{KH}: \therefore$ if $\mathrm{KC}=\mathrm{AR}$, $C$ gives the medial section of $A B$; and if $K C^{\prime}=K C, C^{\prime}$ is point of external section, corresponding to the negative root of equation (1.).

\section{(Figure 5.)}

Ex. v. Solve $\mathrm{AB} . \mathrm{BC}=\frac{2}{3} \mathrm{AC}^{2} . \quad$ Here $p=\frac{2}{3}, \operatorname{surd}=\sqrt{\frac{11}{3}}=\frac{1}{3} \sqrt{ } 33$. Produce $A B$ both ways. In $A B$ produced take $A K=\frac{3}{2} A M$, $A W=3 A K$, and $A Y=11 A K . \quad \therefore A R=\sqrt{3 \times 11}$ if $\perp A B$ and limited by $\frac{1}{2} \odot$ on $W Y$. Hence if $K C=\frac{1}{3} A R, C$ is the point required; and if $\mathbf{K \mathbf { C } ^ { \prime }}=\mathbf{K C}, \mathbf{C}^{\prime}$ is the point of external section, as in Ex. iv. above.

Thus for a given value of $p$ the surd number $\sqrt{4 p+1}$ though intractable to Arithmetic can always be found by Geometry. In certain cases lower surds should be subsidised: thus for $\sqrt{\mathbf{3 3}}$ (just found by II : 14) we may say $33=5^{2}+(2 \sqrt{ } 2)^{2}$.

Similarly $\quad 21=4^{2}+(\sqrt{ } 5)^{2} ; \quad 181=13^{2}+(2 \sqrt{ } 3)^{2}$.

* In the diagrams $M$ is the mid-point of $A B$ and in Figs. 1, 2, 3, SAK is $\perp \mathrm{AB}$. 
§II.

(Figure 6.)

For the general case of equation (1.), the surd line $\sqrt{4 p+1}$ can be expressed either by $I: 47$, since $4 p+1=(2 \sqrt{ } p)^{2}+1^{2}$, or by II : 14. Thus, by the latter, produce $A B$ both ways till $\mathrm{AK}=$ the unit $=\frac{\mathrm{AB}}{2 p}$ and $\mathrm{BH}=\mathrm{BK}$, so that $\mathrm{AH}=4 p+1$ units. $\therefore \mathrm{AR}=\sqrt{4 p+1}$ if $\perp \mathrm{AB}$ and limited by $\frac{1}{2} \odot$ on $\mathrm{KH}$. Hence if $\mathbf{K C}=\mathbf{K} \mathbf{C}^{\prime}=\mathrm{AR}, \mathrm{C}$ and $\mathbf{C}^{\prime}$ are the required points of section.

(Figure 7.)

Mr G. Duthie suggests a third general construction for eq. (1.). "Produce $\mathrm{AB}$ till $\mathrm{BK}=\mathrm{MB}=\mathrm{MA}$, and take $\mathrm{MH}=\frac{\mathrm{AM}}{p}$ : with centre $K$ and radius $K M$ describe $\odot M Q W$. Finally take $H R=H Q$ the tangent from $\mathrm{H}$. Then if $\mathrm{AC}=\mathrm{MR}, \mathrm{C}$ is the point required."

As shown above, $\mathrm{C}$ and $\mathrm{C}^{\prime}$ are two points in $\mathrm{AB}$ or its production which determine the roots of the original quadratic. Thus, if $\mathrm{AR}=\frac{\mathrm{AB}}{2 p}$, then

(a.), when $p$ is positive, with limits $\infty$ and 0 ,

$$
\mathbf{A C}=\mathbf{A R}(\sqrt{1+4 p}-1) \text {, and } \mathbf{A C}^{\prime}=-\mathbf{A R}(\sqrt{1+4 p}+1) \text {, }
$$

$\therefore$ as $\mathbf{A R}$ grows continuously from 0 to $\infty$, so $\mathrm{C}$ and $\mathrm{C}^{\prime}$ move further apart from $A$ in opposite directions ;

$(\beta$.$) , when p$ is negative, with limits $-\frac{1}{4}$ and $-\frac{1}{\infty}$,

$$
\mathbf{A C}=\mathbf{A R}(1-\sqrt{1+4 p}) \text {, and } \mathbf{A C}^{\prime}=\mathbf{A R}(1+\sqrt{1+4 p}) \text {, }
$$

$\therefore$ as $\mathrm{AR}$ grows continuously from $-2 a$ to $-\infty$, so $\mathrm{C}$ and $\mathrm{C}^{\prime}$ move in opposite directions further and further apart from $Z$ which is a point in $\mathrm{AB}$ produced positively so that $\mathrm{AZ}=2 a$.

$(\gamma$.$) . At any instant both for \left(a_{\text {. }}\right)$ and $(\beta$.$) , the distance \mathrm{CC}^{\prime}=$ twice the surd-line; and, numerically, $\mathrm{AC}^{\prime}-\mathrm{AC}=2 \mathrm{AR}$. 
We may note also from equation (1.) that generally $\frac{\mathrm{AO}}{\mathrm{AM}}=\frac{\sqrt{4 p+1}-1}{p} ; \quad \therefore \mathrm{AC} \lessgtr \mathrm{AM}$ according as $\sqrt{4 p+1} \lessgtr 1+p$, or as $2 \lessgtr p$ : [Thus $\mathrm{AC}=\frac{1}{2} \mathrm{AB}$ when $p=2 ; \mathrm{AO}>\frac{1}{2} \mathrm{AB}$ when $p<2$ as in Euclid II : 11 and Ex. v. above; $\mathrm{AC}<\frac{1}{2} \mathrm{AB}$ when $p>2$ as Ex. i., ii., iii.],

\section{\$III.}

\section{(Figure 8.)}

To show graphically the variation of the segment $A C$, as obtained by equation (1.), $I$ have placed $P_{1} R_{1} P_{2} R_{2} P_{3} R_{3} \ldots \ldots$ perpendicular to the fixed line $A B$, so that

$$
\mathbf{P}_{1} \mathbf{R}_{1}=\mathbf{A R}_{1}\left(\sqrt{ }^{/ 5-1)}, \quad \mathbf{P}_{9} \mathbf{R}_{2}=\Delta \mathbf{R}_{2}(\sqrt{ } 9-1), \quad \mathbf{P}_{3} \mathbf{R}_{3}=\right.\text { etc., etc., }
$$

and generally $\mathrm{PR}=\mathrm{AR}(\sqrt{4 p+1}-1)$, where $\mathrm{PR}=\mathrm{AC}, \mathrm{AR}=\frac{\mathrm{AB}}{2 p}$

$$
\begin{aligned}
& \therefore \quad \mathrm{PR}+\mathrm{AR}=\mathrm{PS}=\mathrm{AR} \sqrt{4 p+1}=\frac{\mathrm{AS}}{\sqrt{2}} \sqrt{4 p+1} \\
& \left.\begin{array}{rl}
\therefore \quad 2 \mathrm{PS}^{2} & =\mathrm{AS}^{2}(4 p+1) \\
& =\mathrm{AS}(2 a \sqrt{ } 2+\mathrm{AS})
\end{array}\right\} \text { since } 4 p=\frac{2 a}{\mathrm{AR}}=\frac{2 a \sqrt{ } 2}{\mathrm{AS}}, \\
& =\text { AS. A'S, }
\end{aligned}
$$

Hence for $P$, any point in the locus, the square of PS has a constant ratio to the rectangle AS. $A^{\prime} S$, and that is the geometrical property of a Hyperbola having A'OASE as a diameter and PS an ordinate to it. Thus $P S=S Q, F A D$ is a tangent at $A$, $F^{\prime} A^{\prime}$ another at $A^{\prime}$, and $O$ is the centre of the curve.

With reference to the original problem, $P S$ (or $S Q$ ) is the surd-line $\sqrt{4 p+1}, A R$ (or $R S$ ) the unit-line, and the two roots of equation (1.) are $P R$ and $R Q$. If, for example,

$$
\left.\begin{array}{rl}
p=1, \mathrm{SP} & =\mathrm{SQ}=\sqrt{ } 5 \\
\mathrm{PR} & =\sqrt{ } 5-1=\mathrm{AC}, \text { internal segment } \\
\mathrm{RQ} & =-\sqrt{ } 5-1=\mathrm{AO}, \text { external segment }
\end{array}\right\} \text { and Euclid II }: 11 .
$$

The ordinate at $\mathbf{P}^{\prime}$ if produced upward to meet the branch $P^{\prime} V^{\prime} A^{\prime}$ in $Q^{\prime}$, and downward to meet $A B$ in $R^{\prime}$, gives $P^{\prime} R^{\prime}=A C$ and $Q^{\prime} \mathbf{R}^{\prime}=A C^{\prime}$, for $p$ negative. The Euclidian solution therefore of this case must place $C$ and $O^{\prime}$ in $A B$ produced through $B$, as already shown, §II. 
$\S I V$.

The Cartesian equation to this Hyperbola is at once derived from equation (3.) thus, choosing OFG as the axis of $x$,

$$
\begin{aligned}
& 2 \mathrm{PS}^{2}=\mathrm{AS} . \mathrm{A}^{\prime} \mathrm{S}=(\mathrm{OS}-a \sqrt{ } 2)(\mathrm{OS}+a \sqrt{ } 2) \\
&=\mathrm{OS}^{2}-2 a^{2} \\
& \therefore \quad 2(x+y)^{2}\left.=2 x^{2}-2 a^{2}\right\} \text { putting } x+y \text { for PS and } x \sqrt{ } 2 \text { for } \mathrm{OS} . \\
& \therefore \quad y^{2}+2 x y+a^{2}=0 . \quad . \quad . \quad . \quad . \quad \text { (4.) }
\end{aligned}
$$

The form of this equation shows that one asymptote is parallel to the axis of $x \therefore$ OFG is that asymptote. But $y^{2}+2 x y=y(y+2 x)$, $\therefore y+2 x=0$ is the other asymptote, viz., the line OD. Thus V'OV bisecting the angle FOD is the transverse axis of the Hyperbola, $\mathrm{V}$ and $\mathrm{V}^{\prime}$ are the vertices.

Finally, referring the curve to its own axes, equation (4.) becomes

$$
\frac{x^{2}}{\sqrt{5+1}}-\frac{y^{2}}{\sqrt{ } 5-1}=\frac{a^{2}}{2}, \quad \text { or }\left(\frac{x}{h}\right)^{2}-\left(\frac{y}{k}\right)^{2}=1 \text {, }
$$

where

$$
\begin{aligned}
& h^{2}=O \nabla^{2}=\frac{a^{2}}{2}(\sqrt{ } 5+1)=2 a^{2} \cos \frac{\pi}{5} \\
& k^{2}=O W^{2}=\frac{a^{2}}{2}(\sqrt{ } 5-1)=2 a^{2} \cos \frac{2 \pi}{5}
\end{aligned}
$$

Thus $h k=a^{2}=\frac{1}{4} L L^{\prime}$, where $\mathrm{L}$ is the lat. rectum of the curve, and $L^{\prime}$ that of its conjugate. Hence

$$
\mathrm{L}=4 k \cos 72^{\circ} \text {. }
$$

A curious property therefore of our Hyperbola is that

$$
\frac{\mathrm{L}}{\mathrm{WW}^{\prime}}=\frac{\mathrm{WW}^{\prime}}{\overline{\nabla V^{\prime}}}=\frac{\nabla \mathrm{V}^{\prime}}{\mathbf{L}^{\prime}}=2 \cos 72^{\circ}
$$

In other words, an isosceles $\Delta$ satisfying Euclid IV :10 is found by taking the two terms of any one of those three fractions, and making the numerator the base.

Another singular property, easily deduced, is that

$$
r^{\frac{2}{3}}+a^{\frac{2}{3}}=(4 \mathrm{~L})^{\frac{2}{3}}, \quad \text {. . . . . }
$$

where $r=$ radius of curvature at the extremity of $L$ the latus rectum. 